\title{
Application of ozone ultrafine bubble water for cleaning and sterilizing the dialysis circuit
}

\author{
Yukiko Nakahara ${ }^{{ }^{*}}$, Kazutoku Miyazaki ${ }^{2}$, Junji $\mathrm{Nakao}^{3}$, and Shijie Zhu ${ }^{4}$ \\ ${ }^{1}$ Department of Medical Engineering, Junshin Gakuen University, Fukuoka, Japan \\ ${ }^{2}$ Hakujinkai Hospital, Fukuoka, Japan \\ ${ }^{3}$ Tosslec Co., Ltd., Kyoto, Japan \\ ${ }^{4}$ Department of Intelligent Mechanical Engineering, Fukuoka Institute of Technology, Fukuoka, Japan
}

\begin{abstract}
The dialysis circuit was washed by the ultrafine bubble (UFB) water and ozone UFB water and sodium hypochlorite aqueous solution. The effect of washing time, ozone concentration of UFB water, influence of UFB water on artificial dialysis equipment, etc. were investigated. The cleaning and sterilizing effect of ozone UFB water passed through the filter for artificial dialysis was verified. It was confirmed that there were no colony bacteria in the circuit which was washed by the ozone UFB water for 1 hour. Ozone UFB water has the same bactericidal effect as sodium hypochlorite. As an alternative of sodium hypochlorite, we are expecting its application to the cleaning and sterilization of ozone UFB water for dialysis circuit.
\end{abstract}

\section{Introduction}

In Japan, the number of chronic dialysis patients are increasing in trend, and already over 320,000 [1]. The artificial dialysis equipment is used for treatment of chronic dialysis. As Detergents, sodium hypochlorite and peracetic acid are used for cleaning and sterilizing dialysis circuit. Sodium hypochlorite can remove and disinfect organic matter such as protein, fat, carbohydrate, and the peracetic acid can remove bacteria in dialysis circuit. However, conventionally used detergents have corrosiveness and irritating odor, and they have adverse effects such as detrimental effects when adhering to the skin [1]. In recent years, research on fine bubble technology was thriving and applied in industrial field, agriculture field, fishery field, daily life. The bubble diameter of micro bubbles is visible at $100 \mu \mathrm{m}$ or less. It has a feature that it disappears in water after rising very slowly. Ultrafine

*Corresponding author: nakahara@junshin-u.ac.jp bubble is a bubble smaller than micro bubbles and has a maximum peak at about 100 to $200 \mathrm{~nm}$. It is not visible, has a characteristic that it remains in water for a long time (a few months) [2] and shows Brownian motion. Typical effects of fine bubbles include physical adsorption, cleaning sterilization effect, gas dissolution effect, gas inclusion effect, and physiological activity effect [2-10]. We focused on the gas inclusion effect and attempted to clean the dialysis circuit using ozone ultrafine bubble water.

The purpose of this study was to evaluate the cleaning and sterilizing effect of the ultrafine bubble (UFB) water with or without ozone in the artificial dialysis equipment.

\section{Materials and experimental methods}

The experimental equipment included an RO equipment which manufactured by Nipro Co., Ltd., an ozone ultrafine bubble generator (HMB - 0 Z01) manufactured 
by Tosslec Co. Ltd., an endotoxin measuring instrument (Toxinometer Mini) manufactured by Wako Pure Chemical Industries. The verification sample was the used dialysis equipment circuit, provided by Hakujinkai Hospital. The experimental instruments were a $3.9 \mathrm{~L}$ polypropylene washing vessel. A bacterial culture was a $37 \mathrm{~mm}$ quality monitor (GN-6 Metricel) of Nippon Pole Co., Ltd., and a liquid culture medium of M-TGE broth was used. The soiled dialysis drain pipe (hose) was incorporated into a washing container, and the water around it was taken out and used as a test substance. The cleaning time was from 0.5 hour to 24 hours.

\section{Results and Discussion}

The soiled dialysis circuit was washed with ozone UFB water and tap water for $1 \mathrm{~h}$, respectively. The ozone concentration of ozone UFB water was about 10.00 $10.80 \mathrm{mg} / \mathrm{L} .50 \mathrm{~mL}$ of the test substance was collected and cultured at $37^{\circ} \mathrm{C}$, for 7 days using a $37 \mathrm{~mm}$ quality monitor. As shown in Fig. 1, when washed with ozone UFB water, colony bacteria were not observed. On the other hand, in the case of washing with general tap water, as shown in Fig. 2, colony bacteria were observed after washing for 1 hour.

\subsection{Effect of ozone on cleaning effect}

The cleaning effect of oxygen UFB water and ozone UFB water was compared. The concentration of oxygen was $99.999 \%$. The ozone concentration was about 10.00 - $10.80 \mathrm{mg} / \mathrm{L}$. Oxygen UFB water was placed in a washing container, soiled dialysis piping was placed in a washing container and washed for 1 hour. As shown in Fig. 3, colony bacteria were observed. Comparison of the number of colonies after washing for 1 hour by tap water, oxygen UFB water and ozone UFB water respectively was shown in Fig. 4. It was confirmed that there was a cleaning and sterilizing effect only by use of ozone UFB water.

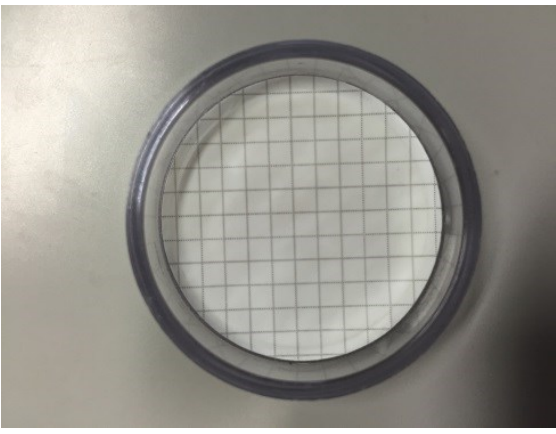

Fig. 1 Cleaning with Ozone UFB for 1 hour.

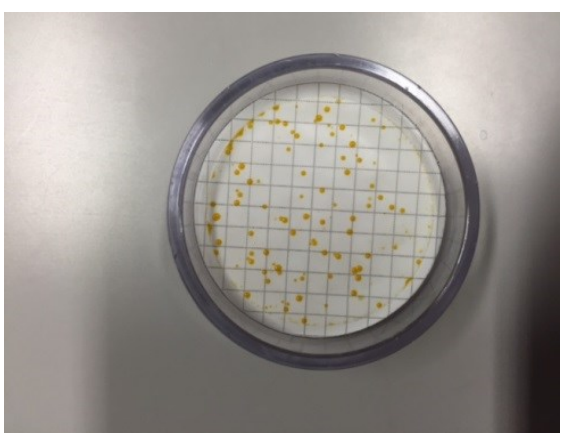

Fig. 2 Cleaning with tap water for 1 hour.

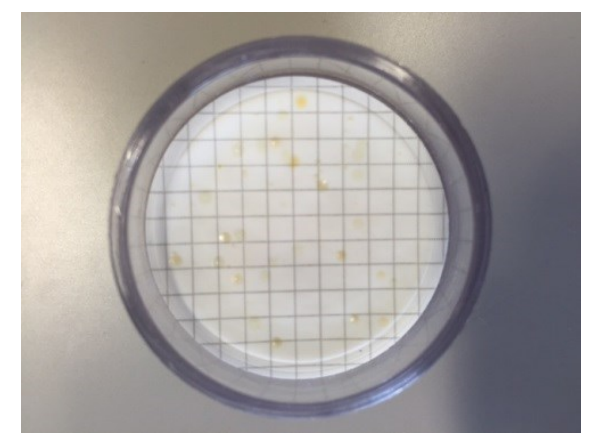

Fig. 3 Cleaning with UFB for 1 hour.

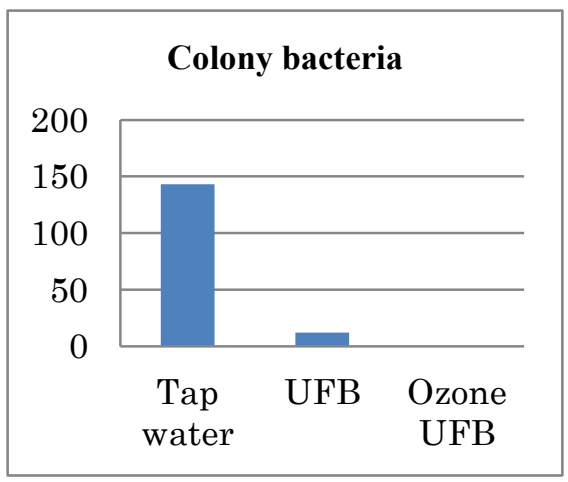

Fig. 4 Comparison of the number of colonies. 


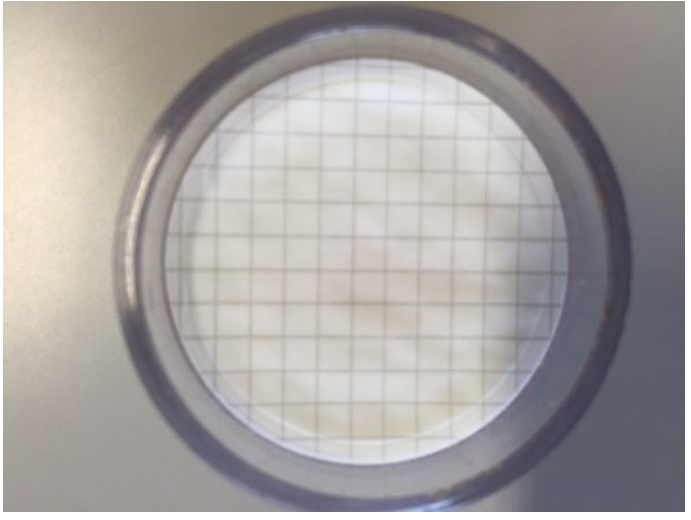

Fig. 5 Cleaning with 300 ppm sodium hypochlorite water for 1 hour.

\subsection{Comparison of cleaning effect of sodium} hypochlorite and ozone UFB water

The effect of washing time, ozone concentration of UFB, influence of UFB water on artificial dialysis equipment, etc. were investigated using sodium hypochlorite of ECO-200, diluted 200 times with general tap water, with the concentration of sodium hypochlorite aqueous solution of $300 \mathrm{ppm}$. The cleaning and sterilizing effect of ultrafine bubble water passed through a filter for artificial dialysis was verified.

\section{Conclusion}

The bactericidal effect of ozone ultrafine bubble water was obtained. As an alternative of sodium hypochlorite, we are expecting its application to the cleaning and sterilization of ozone UFB water for dialysis circuit and artificial dialyzer.

\section{References}

1. E. Masakane et. al., Journal of Japanese Society for Dialysis Therapy, Vol. 50, No. 1, 1-62 (2017)

2. Y. Kanai, K. Terasaka, S. Fujioka and K. Suzukawa, J. Chem. Eng. Japan, Vol.50 (2), 86-93 (2017)

3. K. Terasaka, J. Nitta, H. Fukuda, M. Iwahashi, T. Goshima, S. Fujioka, J. Chem. Eng. Japan, 46 (1), 33-39 (2013)

4. M. Takahashi, E. Izawa, J. Etou and T. Ohtani, Journal of the Physical Society of Japan, 107-10, 2171-2173 (2003)

5. K. Terasaka, Chemical Engineering of Japan, Vol.78, No.9, 580-584 (2014)

6. N. Sumida, $1^{\text {st }}$ International Symposium of Fine Bubbles, (2012)

7. S. Maeda et al., $1^{\text {st }}$ International Symposium on Multiscale Process Engineering (MMPE), Kanazawa, Japan, P. 24-1 (2011)

8. M. Takahashi et al., J. Phys. Chem. B 107, 2171, (2003)

9. N. Sumida, Chemical Engineering of Japan, Vol. 78, No. 9, 589-592 (2014)

10. T. Fujita, Chemical Engineering of Japan, Vol. 78, No. 9, 585-588 (2014) 